\title{
Analyzing the Causes of Rumors in the Context of Public Emergencies: A Literature Review
}

\author{
Hu Jiyuan ${ }^{1, *}$ \\ ${ }^{1}$ Business School, Sun Yat-sen University, Guangzhou, Guangdong, China \\ *hujy53@mail2.sysu.edu.cn
}

\begin{abstract}
Rumors occur in the context of public emergencies, which bring great trouble to the public. Studies of the occurrence of rumors will enable the government to deal with public emergencies more effectively. Although there were many studies related to this field, they are relatively unsystematic. In view of this, this paper will analyze the internal and external causes of rumors and establish a literature review. This paper has important indications for future studies and rumor management in the context of public emergencies.
\end{abstract}

Keywords: rumor, public emergency, cause

\section{突发公共事件背景下谣言成因分析综述}

\author{
胡纪元 ${ }^{1,}$
}

${ }^{1}$ 中山大学管理学院, 广州, 广东, 中国

hujy53@mai12.sysu.edu.cn

\section{摘要}

在发生突发公共事件时，各种谣言满天飞，给民众带来极大的困扰。如果能够了解谣言产生的原因， 从而采取相应的措施, 就可以净化民众文化环境, 使大家集中精力更科学有效地应对突发公共事件。 虽然以往对于谣言成因的研究很多, 各种内外部影响因素均有涉猎, 但比较杂乱、不成体系。鉴于此, 本文按照内部因素和外部因素两个方面, 对谣言的产生进行仔细的分析, 并写作综述。本研究结果对 于之后谣言产生的研究方向和突发公共事件谣言的管理有重要的启发。

关键词: 谣言，突发公共事件，成因

\section{1.引言}

2020 年年初, 新冠状病毒在中国爆发。与此同时, 新冠疫情相关谣言在社会中大范围地传开, 人们一时间 人心惶惶。

谣言究竟为何会产生? 为何在疫情期间尤其严重? 针对谣言产生的研究可以很好地解释以上两个问题。

\section{2. 研究述评}

对于谣言如何产生的研究最早是由 Allport 与 Postman ${ }^{[1]}$ 展开, 他们提出了一个重要公式, 即 $R=I$ (Importance) $*$ A (Ambiguity). 他们的研究表示, 谣言 的产生有两个基本条件, 故事的主题对传谣者和听谣者 有某种重要性和真实的事实被某种模糊性所掩盖。

此后, 对于谣言产生的研究层出不穷, 可以分为外 部因素和内部因素两个角度。

\section{1. 外部因素研究}

从外部因素研究谣言产生的原因主要从特定情景、 媒介以及政府的角度入手。

\section{1. 1. 情境因素}

早期的研究主要是集中在谣言产生的背景, 即谣言 在何种情境下会出现。Knapp ${ }^{[2]}$ 指出谣言在社会压力时期 盛行。一些学者将突发公共事件与谣言的研究联系了起 来, 认为突发公共事件由于其重大性, 天然地造成了不 实信息的产生。陈万怀 ${ }^{[3]}$ 就认为, 谣言在战争、社会变 更、自然灾害如地震、疫情等突发公共事件出现时会大 量产生。兰月新 ${ }^{[4]}$ 对于突发事件这个谣言产生的社会情 境作出了阶段区分，将突发事件分为事件发生、网络與 情产生和传播、政府與情引导、事件平息等阶段，并与 网络舆情的潜伏期、明显期、高潮期和衰退期所对应, 充分表明了社会情境对于谣言产生与传播的影响。 


\subsection{2. 政府以及媒介角度}

此后的一些研究则不仅仅停留在了谣言产生的背 景层面, 而试图从政府以及媒介的角度, 找到不实信息 产生的原因。马广海 ${ }^{[5]}$ 就认为政府公信力下降是造成政 治谣言的主要原因。周裕琼 ${ }^{[6]}$ 也从政府、媒介的责任入 手, 认为官方信息的缺失, 使得群众需要通过造谣来挖 掘事实，从而导致了谣言的产生。

\section{2. 内部因素研究}

大部分谣言产生相关的研究是围绕个人层面展开 的，对于处于突发公共事件下个体的认知、情绪和动机 等进行研究。

本文认为, 认知 - 情绪/动机 - 行为是一个完整的可 以阐明谣言产生机制的认知过程, 人们对于情境的认知 导致了情绪以及动机的形成, 这些因素进而促使了人们 相信、传播谣言。因而, 下面的文献综述将从认知开始, 再到情绪、动机相关的研究。

\subsection{1. 认知}

人们处于一特定的社会情境下, 由于个人特质等的 不同, 会对事件形成独有的认知。对情境的认知与外部 因素中的情境因素不同, 前者是主观的, 受个人因素的 影响; 后者则具备客观性。

Allport ${ }^{[7]}$ 的谣言公式 $\mathrm{R}=\mathrm{I}$ (Importance) $* \mathrm{~A}$ (Ambiguity) 就表明了情境需具备模糊性和重要性等两 大特点, 即人们若同时感知到情境中的这两大特性, 则 谣言容易产生。Faye ${ }^{[8]}$ 对于 Allport 的理论进行了补充, 认为当个体处于模糊、不确定的情境中会焦虑感较强, 若同时出现对个体重要的话题, 谣言就会容易产生。 Difonzo 等 $^{[9]}$ 在之前研究的基础上提出, 不信任的环境 以及正式交流渠道的惯乏也是谣言的温床。

可以看到, 以上的研究结果认为, 情境的模糊性、 重要性、不确定性以及不信任性都是谣言的摇篮, 而这 些因素并不是完全客观的, 会受到个人性格特征、接受 消息渠道等一系列的影响, 具备主观性。因而以上的因 素不仅是谣言产生的情境规律, 更是人们对于情境的认 知特点。

\subsection{2. 情绪}

研究者在研究内部因素中谣言产生的心理基础时, 大多是从情绪的角度出发。早在 1935 年, Prasad 在研 究 1934 年印度地震期间的谣言时发现, 谣言是一个群 体对一个影响到整个群体利益的高度情绪化的情况的 反应, 由于谣言是在群体情境中产生的, 其特点一定程 度上取决于群体的心态 ${ }^{[10]}$, 这便是在一定程度上肯定了 谣言的产生与人的情绪有关。在此后的研究中, Prasad 又注意到了个体心理上的好奇、焦虑、探索欲与不安感,
注意到了这些情绪是相同的, 以至于在群体中发展出了 情绪的整体感 ${ }^{[11]}$ 。应该说, Prasad 虽没有专门研究谣言 产生的情绪基础, 但他在丰富的人类感受基础上, 提出 了情节情感性对于谣言的产生所具有的决定性影响。

Prasad 对于谣言产生的情绪基础研究处在一个相 较广的层面, 包括了多种情绪感受。此后对于谣言产生 的情绪研究则狭隘了很多, 多聚焦在焦虑感这一种情绪 体验上，而忽视了好奇、不安等其他情绪。

Festinger 将预测重大灾难的谣言称为 “证实焦虑” 的谣言。他进一步推测, 人们对于灾难的未知与由灾难 引起的恐慌是一种认知失调, 而谣言提供了与害怕相协 调的认知, 因此谣言可以被称为 “产生害怕” 的谣言 ${ }^{[12]}$ 。 罗斯洛则在 Prasad 和 Allport 等的研究基础上进一步 推进, 为谣言公式加入了第三个因素: 焦虑, 并衍生出 情境焦虑、人格焦虑和谣言焦虑 ${ }^{[133}$ 。此后, 一些学者对 于这三种不同类型的焦虑与谣言传播的关系开展了进 一步研究。

也有少量学者注意到了不实信息产生涉及的其他 情感因素。李智 ${ }^{[14]}$ 认为谣言的产生是基于一种恐惧和孤 立的情感状态, 人们基于心理上的不安, 会产生一种消 灭信息真空性的强烈欲望。王锁明 ${ }^{[15]}$ 则囊括了一系列的 情感因素, 如娱乐, 猎奇, 怀疑, 较真, 恐慌, 焦躁, 盲从等。

\subsection{3. 动机}

还有一类学者探究动机方面的原因。

郑保卫等 ${ }^{[16]}$ 将谣言的产生归因于信息控制、归因心 理和次级控制等心理机制。Difonz ${ }^{[17]}$ 则认为谣言是出 于事实调查 (fact-finding), 建立关系 (relationshipbuilding) 和自我提升 (self-enhancing) 这三种深层动 机, 其中事实调查是谣言产生的动机, 而建立关系、自 我提升属于谣言的传播方面的动机。

\section{2. 4. 多角度对内部因素的研究}

此前对于谣言产生原因的内部因素研究将情绪与 认知、动机割裂开来, 分开研究。然而, 需知谣言的产 生所涉及的心理机制十分复杂, 认知、情绪以及动机间 具有难以分割的相互联系, 将三者割裂研究并不可取。

因此, 郭小安 ${ }^{[18]}$ 将情绪与动机一同研究, 并认为个 人情感如焦虑、不安、压抑以及个人理性（有限理性、 不良企图、趋利弊害）会造成谣言的产生。他还在 Difonzo ${ }^{[19]}$ 对谣言产生的动机因素研究的基础上进一步 加深了研究, 将 Difonzo 的动机因素与情感联系起来, 进一步认为谣言的产生是出于人们恐惧和不安的感受, 报复、欲望、利益等动机的释放以及谣言是人们期望的 另类表达 ${ }^{[20]}$ 。

可以看到, 即使有些学者考虑到了将情绪、认知与 动机放在一起来研究谣言产生的原因, 他们的研究仍是 分散的, 即没有一个完善的机制将谣言产生过程中的各 
种因素囊括在一起。

少数学者做出了尝试。张赛宜 ${ }^{[21]}$ 在研究中试着搭建 框架, 尝试使用 $\mathrm{ABC}$ 理论将认知与情绪联系起来, 研究 群体性事件的形成过程。杨慧琼 ${ }^{[22]}$ 等则从人们的情绪、 认知以及行动多个视角来研究食品安全谣言的产生, 并 认为人们对于谣言的认知、情境情绪以及谣言情绪进一 步导致了行为意图, 将谣言产生中的因果逻辑利用情绪 框架清楚地叙述了出来。

\section{3. 外部环境下的内部因素研究}

大量的研究对于谣言的产生研究都是将内部与外 部因素分开, 各自研究。然而, 谣言的产生具有很强的 情境性, 群众的心理情况也很大一部分依托于情境的变 化, Allport 的谣言公式中强调的情境重要性以及模糊 度也是群众个人感知的结果, 想要将内部与外部层面割 裂、单独研究对于现实生活的参照意义不大。因而, 一 些学者从内部与外部层面上一同对于谣言的产生进行 研究。

杜骏飞 ${ }^{[23]}$ 认为, 流言的出现是官方信息的缺失造成 了群众对于信息的极度渴求的外在表现。当受众渴求信 息的需求未被满足, 便会产生流言。他将流言产生的因 果逻辑阐述的很清楚。常建等 ${ }^{[24]}$ 认为, 有效信息的不足 导致群众信息饥渴以及刺激性谣言对于受传者精神需 求的满足均导致谣言的产生。霍良安等 ${ }^{[25]}$ 认为, 突发事 件导致人们对于信息的渴求异于平常, 若政府和媒体对 信息的扩散不加以透明化、详细化、控制化, 就会导致 谣言的产生。也有研究者从内压和外压两个角度来研究 谣言的产生, 基于谣言是由情绪压力产生的, 再进一步 阐述情绪合意下的共同体生成内压, 而对话缺失的情绪 蓄积生成外压, 在内压和外压的共同作用下, 产生激变 一一情绪转向下生成谣言 ${ }^{[26]}$ 。

一些研究甚至搭建了研究框架, 将外部与内部因素 纳为一体, 巧妙地运用一个解释机制来阐明谣言的产生。

Kapferer ${ }^{[27]}$ 建立起框架结构, 利用官方传播者是否 予以传播以及受传者的信息需求是否得到满足两个变 量, 判断谣言的发生区 (如图 1)。

\begin{tabular}{|c|c|c|}
\hline & $\mathrm{A}$ 官方传播者予以传括 & B. 官方俊指者不予传括 \\
\hline 1. 受传者的信息需求末满足 & AL. 流言浀在发生区 & B1. 流言高发区 \\
\hline 2 受传者的信息需要得到满足 & A2 流言非发生区 & B2 㳬言易发生区 \\
\hline
\end{tabular}

图 1 谣言发生区

黄文义等 ${ }^{[28]}$ 则使用马斯洛需求理论, 表明安全需要 是最基础的需要之一, 突发事件会给群众一种 “失控” 的感觉, 引起人们不同程度的焦虑和不安。而谣言作为 一种信息的传递方式, 正好满足了人们寻求稳定的心理 需求。闵庆飞等 ${ }^{[29]}$ 则是建立了一个环境因素影响群众的 心理从而引发谣言行为的模型 (如图 2), 说明了环境中 的不同因素分别会对群众的认知以及心理状态产生怎 样的影响, 而这些影响又为何会导致谣言行为。

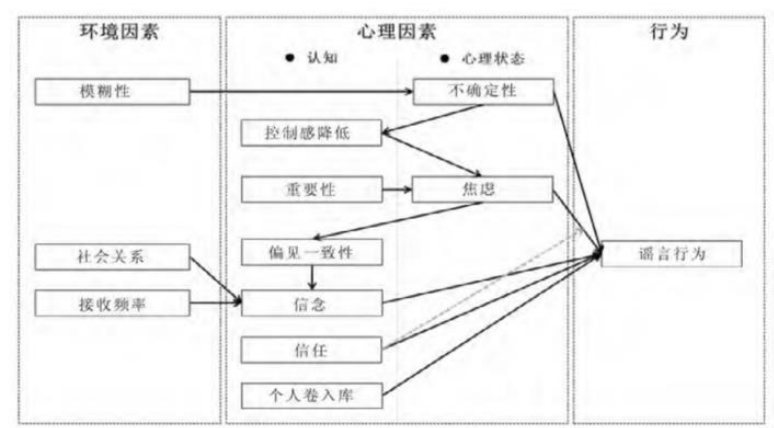

图 2 谣言模型

\section{3.总结与展望}

由以上分析可以看到, 国内外均有大量针对不实信 息产生的研究, 从外部因素的研究上来说, 从研究谣言 产生的情境因素，到包括政府以及媒介这些外部因素对 于谣言的影响; 从内部因素的研究上来说, 经历了单一 地研究情绪、认知、动机等过渡到多角度地对于内部因 素进行研究，并尝试使用相关的理论进行整合、归纳; 更有学者意识到了个人心理与情境的不可分割性, 针对 内部以及外部因素共同研究, 并试图搭建模型对于谣言 的产生机制进行更好的解释 (如图 3 研究发展脉络示 意图)。

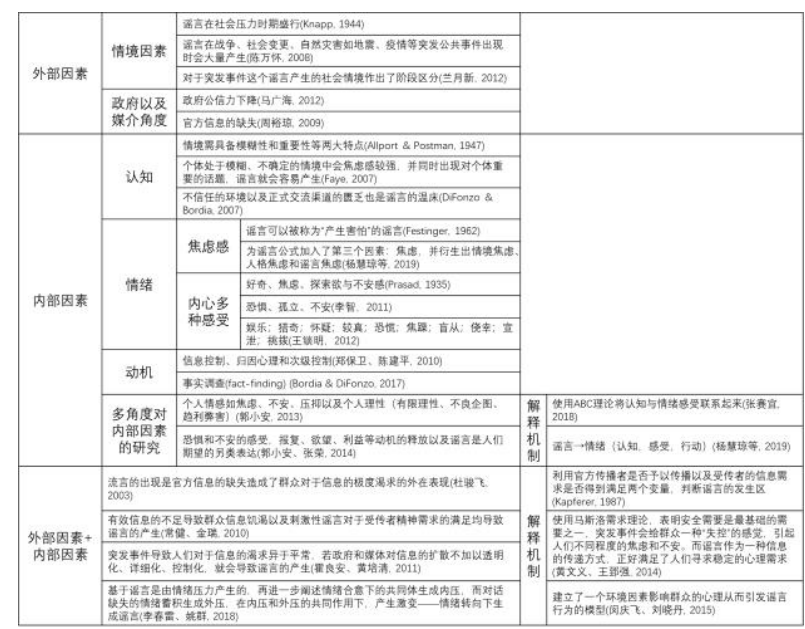

图 3 研究发展脉络示意图

国内的研究多是在特定的事件或环境下对于谣言 成因进行分析, 并且大多是从政府部门监管、法律法规 处罚以及媒介的责任等方面展开的, 从心理因素层面进 行探讨的并不多 ${ }^{[30]}$ 。即使有一些对于谣言产生心理基础 的研究, 也大多是定性研究。国外的研究开始较早, 但 研究问题以及研究结果雷同, 大多是针对某单一情绪感 受对于不实信息的影响研究, 或是引用了A1lport 的著 名公式, 并且忽略了外部因素对于谣言的影响。不过, 国外学者善于采用实验来证明假设, 而国内的学者则更 倾向于将问题置于某社会情境下。虽然真实场景具有更 强的生态学效度, 但对变量的控制并不严格 ${ }^{[31]}$ 。 
在此后的研究中, 研究者应在研究中囊括认知、情 绪以及动机等一系列内部因素, 并将心理因素置于外部 环境下，利用框架或理论探讨内、外部因素是如何互相 影响的。研究者应弥补国内目前对于定性研究的不足, 采用实验的方法, 严谨的推出结果, 从而对于预测突发 事件中谣言的产生情况，更好地预防、疏导谣言。

\section{REFERENCES}

[1] [7] Allport, G. W., \& Postman, L. (1947). The psychology of rumor. Oxford, England: Henry Holt.

[2] Knapp, R. H. (1944). A psychology of rumor. Public opinion quarterly, 8(1): 22-37

[3] Chen, W. H. (2008). Cognitive and Digestion of the Internet Rumors from the Communication Perspective. Press Circles, 06: 50-51

[4] Lan, Y. X. (2012). Propagation Model of Emergency Network Rumor. Library and Information Service, 56(14): 57-61

[5] Ma, G. H. (2012). Group Events: An Analysis of the Social Mentality in the Process of China's Social Transformation. Journal of Ocean University of China(Social Sciences), 06:71-79

[6] Zhou, Y. Q. (2009). Rethinking the Social Functions and Communication Principles of Rumors Based on Literature Review and Empirical Studies. Journal of International Communication, 08:51-54

[8] Faye, C. (2007). Governing the grapevine: The study of rumor during World War II. History of psychology, 10(1):1

[9] DiFonzo, N., \& Bordia, P. (2007). Rumor psychology: Social and organizational approaches.: American Psychological Association. American Psychological Association (APA), Washington

[10] Prasad, J. (1935). The psychology of rumour: A study relating to the great Indian earthquake of 1934. British Journal of Psychology. General Section, 26(1):1-15

[11] Prasad, J. (1950). A comparative study of rumours and reports in earthquakes. British Journal of Psychology. General Section, 41(3-4):129-144

[12] Festinger, L. (1962). A theory of cognitive dissonance (Vol. 2): Stanford university press

[13] Rosnow, R. L., Esposito, J. L., \& Gibney, L. (1988). Factors influencing rumor spreading: Replication and extension. Language \& Communication, 8(1):29-42

[14] Li, Z. (2011). Rumor, Gossip and Legend. Journal of Beijing Administrative College, 02:115-119
[15][30] Wang, S. M. (2012). Analysis of causes of network rumors based on social psychology. Observation and Ponderation, 09:44-47

[16] Zheng, B. W., \& Chen, J. P. (2010). The transmission and blocking mechanism of rumors. Chinese Journalist, 05:42-43

[17][19] Bordia, P., \& DiFonzo, N. (2017). Psychological Motivations in Rumor Spread. Rumor Mills. Routledge, pp.87 102.

[18] Guo, X. A. (2013). The Political Incentive of Cyber Rumors: Integration of Theory and Chinese Experience. Wuhan University Journal (Humanity Sciences). 03:120124

[20] Guo X. A., \& Zhang, R. (2014). Three aspects of rumor psychology. Journal of Tianjin Administration Institute. 03:41-50

[21] Zhang, S. Y. (2018). A Social-psychological Analysis of the Forming Process of Group Events. Journal of Political Science and Law. 03:114-121

[22] Yang, H. Q., Que, Y. L., \& Ma, X. Y. (2019). Emotion mechanism behind rumor transmission: based on research of rumors in food industry. Southeast Communication. 08:94-100

[23] Du, J. F. (2003). The Spread of Gossip: A Mass Media Analysis of the Spread of SARS-related News. Journal of Nanjing University (Philosophy, Humanities and Social Science). 05:116-124

[24] Chang, J., \& Jin, R. (2010). Analysis of function, transmission, and prevention of rumors in public conflicts. Tianjin Social Sciences. 06:65-69

[25] Huo, L. A., \& Huang, P. Q. (2011). The Study of Rumor Diffusion in Emergencies $\longrightarrow$ A Case of Earthquake in Japan. Journal of Intelligence. 10:77-82

[26] Li, C.L., \& Yao, Q. (2018). A Rumor Propagation Study of "Background of Emotion". Journal of Guangzhou University (Social Science Edition). 10:46-50

[27] Kapferer, J. (1987). Rumors. RECHERCHE, 18(187): 468-475

[28] Huang, W. Y., \& Wang, Z. Q. (2014). Analysis of the transmission and government governance mechanism of online rumors in the transitional period. Journal of Chinese Academy of Governance. 03:107-111

[29] Min, Q. F, \& Liu, X. D. (2015). A Review of Rumor Study Based on Media Evolution. Journal of Intelligence. 04:104-109

[31] Peng, X. Z., Cui, F., Jiao, C., \& Li, H. (2018). Factors Influencing Rumor Transmission: Characteristics of Circumstances, Contents, Transmitters and Recipients. Journal of Psychological Science. 04:916-921 